Competing interests None.

Keywords Coeliac disease, Elderly.

\section{PTU-045 COELIAC DISEASE IN THE ELDERLY}

doi:10.1136/gut.2011.239301.173

G K T Holmes, ${ }^{1 *}$ F Moor ${ }^{1}{ }^{1}$ Gastroenterology, Derby Hospitals Foundation Trust, Derby, UK; ${ }^{2}$ Dietetic, Derby Hospitals Foundation Trust, Derby, UK

Introduction Coeliac disease (CD) is increasingly diagnosed in the elderly. The aim of the study was to determine the number of patients with CD diagnosed in later life, their mode of presentation and length of symptoms in a single centre 1958-2008.

Methods The diagnosis of CD was based on characteristic appearances of small intestinal mucosa. Symptoms and length of history at presentation of CD were documented. Extensive efforts were made to identify all patients in the area served by the Derby hospitals. Sources of information included the hospital diagnostic index, histopathology records, dermatitis herpetiformis clinic, immunology laboratory, dietetic department and membership records of the Coeliac Society (Coeliac UK). Since 1978, patients were followed prospectively in a weekly CD clinic by GKTH. Information was stored on an Access data base.

Results Between 1958 and 2008, 1315 patients with CD were diagnosed by small intestinal biopsy. Of these, 874 were female and 441 male giving an overall female to male ratio of 1.98:1. 377 (29\%) were diagnosed over the age of 60 years; 265 (20\%), 168 (13\%), 93 (7\%), $46(3.5 \%), 9(0.7 \%)$ and $1(0.08 \%)$ were diagnosed over the ages of $65,70,75,80,85$ and 90 years respectively.

Of 265 patients diagnosed over the age of 65 years 127 (48\%) had anaemia, 88 (33\%) diarrhoea and 83 (32\%) weight loss. $107(40 \%)$ presented with non-specific symptoms or other complaints. 57 (22\%) patients had anaemia alone and 20 (8\%) diarrhoea alone. Of note only 57 cases (22\%) presented with a combination of weight loss and diarrhoea and 22 (8\%) a combination of weight loss, diarrhoea and anaemia. The average duration of symptoms prior to diagnosis was 40 months (spread 0-720 months). 44 and 32 patients experienced symptoms for over 60 and 120 months respectively, before diagnosis.

Conclusion Almost one third of new diagnoses of CD are made in those over the age of 60 years. The diagnosis is easily missed because only a minority have combinations of weight loss, diarrhoea and anaemia regarded as typical symptoms of CD. Anaemia as the sole feature is found in a fifth of patients and diarrhoea may be the only symptom. $40 \%$ of cases have non-specific or other presentations. Diagnosis in the elderly is common but is often delayed because presentations may not immediately suggest the diagnosis of CD. 